\title{
Fabrication additive et besoins en contrôle
}

\author{
Anne-Françoise Obaton ${ }^{1}$, Alain Bernard ${ }^{2,3}$, Georges Taillandier ${ }^{3}$ et Jean-Marc Moschetta ${ }^{1}$ \\ ${ }^{1}$ LCM, LNE, département dimensionnel, 75015 Paris, France \\ ${ }^{2}$ Ecole Centrale de Nantes, 44321 Nantes, France \\ ${ }^{3}$ AFPR, 92360 Meudon-La-Forêt, France
}

\begin{abstract}
Additive manufacturing is under such media coverage that many manufacturers have recently integrated this process into their production line or investigate this possibility. However, all new application of a technology in the industry reveals needs in metrology and testing. A partnership between the Association Française de Prototypage Rapide and the Laboratoire national de métrologie et d'essais has identified these needs. This paper presents a state of the art on additive manufacturing and metrology and testing requirements generated by the technology.
\end{abstract}

\section{Introduction}

La fabrication additive (FA), jusque-là principalement réservée à la réalisation rapide de prototypes en polymère, connaît une croissance sans précédent. Le fait que des brevets soient tombés dans le domaine public a permis une telle médiatisation de la technologie que tous les industriels, et plus particulièrement ceux concernés par la fabrication de petites séries comme dans l'aéronautique, l'aérospatiale et le médical, s'y intéressent de près.

Créée en 1992, l'Association Française de Prototypage Rapide (AFPR) rassemble les partenaires de la FA. Elle favorise la formation et le transfert de la technologie dans les entreprises. De par son statut, elle est parfaitement au fait des procédés et du marché de la FA. Quant à lui, le Laboratoire national de métrologie et d'essais (LNE) est le laboratoire de référence chargé d'accompagner les industriels dans leur évolution et leurs progrès pour améliorer la compétitivité de leur entreprise en assurant la qualité et la conformité de leurs produits. Un partenariat entre ces deux organismes a permis de recenser les besoins en métrologie et essais que la technologie engendre [1]. L'objectif de ce recensement est de contribuer au développement des références (étalons ou méthodes de mesure), des normes et des bancs de tests nécessaires aux industriels.

Nous présentons, dans ce papier, un état de l'art sur la FA et les besoins en métrologie et essais associés à cette technologie.

\section{La fabrication additive}

\subsection{Définition et principaux intérêts}

La FA regroupe les procédés permettant de fabriquer des pièces à partir de matière première brute transformée, couche après couche, suivant un modèle numérique, et ce sans recourir à de l'outillage. Cette technologie se différencie radicalement de l'usinage qui consiste non pas à créer de la matière mais à en enlever progressivement d'un bloc de matériau pour créer l'objet. Par opposition, on qualifiera l'usinage de méthode soustractive.

La FA ne va pas supplanter les autres techniques de fabrication traditionnelles. C'est une technique complémentaire dédiée à la production de petites séries. Elle permet de réaliser des pièces, monolithiques (suppression des assemblages et des soudures), de géométrie complexe, irréalisables avec les technologies traditionnelles. On citera par exemple les structures alvéolaires appelées «lattices » qui permettent d'alléger des pièces par optimisation topologique ou de favoriser l'intégration des implants dans le corps humain (Figure 1). L'autre principal atout de la FA réside dans le fait qu'elle permet la fabrication en masse de pièces personnalisées.

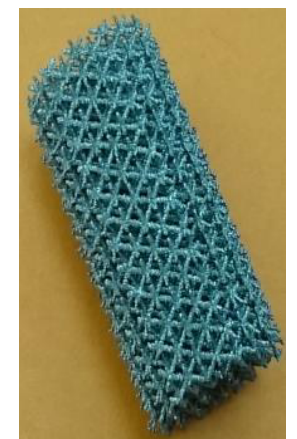

Figure 1. Eprouvette en structure lattice réalisée en FA par Medicrea afin de favoriser l'intégration des implants.

\subsection{Matériau suivant les domaines d'application}

En FA le matériau est élaboré simultanément à la réalisation de la pièce, et ce à partir de matière première

\footnotetext{
a Corresponding author: anne-francoise.obaton@lne.fr
} 
brute se présentant sous forme de liquide, de pâte, de poudre, de filament ou de feuilles. Il s'agira de polymères pour le prototypage et la réalisation de pièces sur lesquelles ne s'exercera aucune contrainte en fonctionnement (Figure 2).

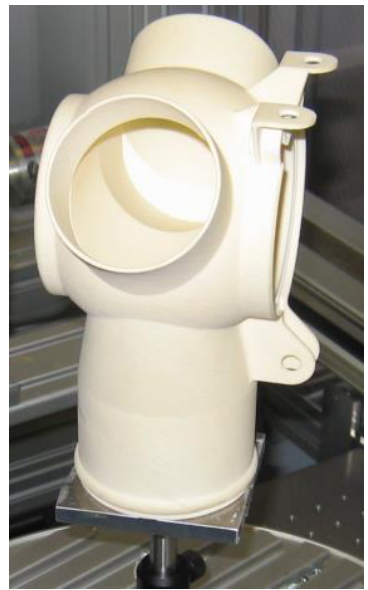

Figure 2. Vanne trois voies en polymère réalisée en FA par Dassault Aviation.

Le secteur automobile utilise beaucoup la FA pour réaliser des parties de ses "concept car» en polymère. Par ailleurs, les machines destinées au grand public ne proposent quasiment que des polymères (acrylonitrile butadiène styrène-ABS-,...).

En revanche, pour la réalisation de pièces fonctionnelles, de haute technicité en petites séries, les secteurs de l'aéronautique et de l'aérospatial privilégieront le métal (inox, chrome-cobalt, aluminium, titane,...). Dans le secteur médical, il s'agira de métal (titane,...) mais également de céramique (hydroxyapatite,...).

Cependant, les secteurs industriels concernés par la production de grandes séries utilisent beaucoup la FA pour du prototypage mais également la réalisation de moules.

Enfin, les matériaux composites commencent à faire leur apparition.

Dans certaines catégories de procédé, la matière première non-utilisée au cours du processus de fabrication peut être recyclée dans les suivants.

\subsection{Catégories et principes des procédés}

Il existe une multitude de procédés de FA, les organismes de normalisation internationaux (ISO/TC 261, ASTM F42) et français (UNM 920) les ont regroupés en sept catégories suivant la forme sous laquelle se présente la matière première et la source d'activation utilisée pour la transformer couche après couche. On les nommera :

1) Photopolymérisation en cuve («vat photopolymerization »);

2) Projection de matière (« material jetting »);

3) Extrusion de matière («material extrusion »);

4) Projection de liant (« binder jetting »);

5) Fusion sur lit de poudre («powder bed fusion $»)$;
6) Dépôt de matière sous flux d'énergie dirigé ( « directed energy deposition »);

7) Stratification de couches («sheet lamination »).

La photopolymérisation en cuve englobe les procédés qui consistent à solidifier sélectivement des photopolymères liquides ou sous forme de pâte avec une source ultraviolette (étroite, de type laser, ou large à travers un masque) ; la projection de matière, ceux qui consistent à déposer sélectivement de fines gouttelettes de photopolymères liquides qui sont ensuite solidifiées avec une source ultraviolette ; l'extrusion de matière, ceux qui consistent à déposer sélectivement un filament de polymère ramolli qui se solidifie ensuite en se refroidissant; la projection de liant, ceux qui consistent à déposer sélectivement de fines gouttelettes de liant sur un lit de poudre (polymère, céramique, métallique) pour la conglomérer; la fusion sur lit de poudre, ceux qui consistent à fusionner sélectivement de la poudre (polymère, céramique, métallique) ; le dépôt de matière sous flux d'énergie dirigé, ceux qui consistent à fondre la matière (métallique) dans une buse au fur et à mesure de son dépôt sélectif ; et la stratification en couche, ceux qui consistent à découper progressivement les couches dans des feuilles (papier, carton, bois) et à les lier.

Dans beaucoup de ces procédés, la pièce doit subir un post-traitement thermique pour parfaire la solidification du matériau ou pour libérer les contraintes mécaniques.

\section{Identification des besoins en contrôle spécifiques à la FA}

\subsection{Contrôles sur la matière première}

En FA, la pièce est fabriquée à partir de matière première se présentant sous forme de poudre, de liquide, de pâte, de filament ou de feuille. La détermination des caractéristiques de ces matières premières permet de produire des pièces dont les propriétés seront connues et prédictibles et ainsi obtenir une bonne répétabilité. Par ailleurs, dans certains des procédés la matière première non-transformée peut être réutilisée dans les cycles de fabrication suivants. En conséquence, la matière première vierge doit être caractérisée mais également la matière première utilisée avant et après recyclage afin de déterminer le nombre de cycles de réutilisation. Enfin, beaucoup d'industriels se plaignent que l'éventail des matières premières utilisables dans les machines est trop restreint. Il faut donc caractériser d'autres types de matière première.

Ces mesures concernent particulièrement les poudres. Cependant, quelques tests peuvent également être pratiqués sur les liquides, les pâtes, les filaments et les feuilles. 


\subsection{Contrôles sur le matériau}

En FA, le matériau est élaboré couche après couche. En conséquence, son élaboration a besoin d'être contrôlée, en temps réel, pour chaque couche, dans la chambre de fabrication, et ultérieurement lorsqu'il est formé. La batterie de tests auxquels il sera soumis après fabrication ne se différencie pas de celle pratiquée sur les matériaux réalisés par des techniques traditionnelles. Cependant, la loi de comportement des matériaux utilisés dans les technologies de fabrication soustractive ou celle des matériaux fabriqués en additif sera différente.

En particulier, pour définir le post-traitement thermique à appliquer au matériau, il faut étudier l'influence de ce post-traitement sur ses propriétés mécaniques.

\subsection{Contrôles sur la pièce finie}

La FA permet de réaliser des pièces de forme complexe, monolithiques. Elle permet notamment de fabriquer des formes gauches (Figure 3), des structures internes et des structures lattices qui nécessitent une inspection en volume et non plus uniquement de surface. Outre la détection de défauts, des méthodes permettant de contrôler la géométrie de la pièce ainsi que ses caractéristiques dimensionnelles sont nécessaires. Qu'il s'agisse de contrôle de surface ou en volume, ces mesures permettent notamment de vérifier la conservation des spécifications géométriques et dimensionnelles définies dans le modèle numérique. A l'heure actuelle, la tomographie à rayons $\mathrm{X}$ est utilisée.

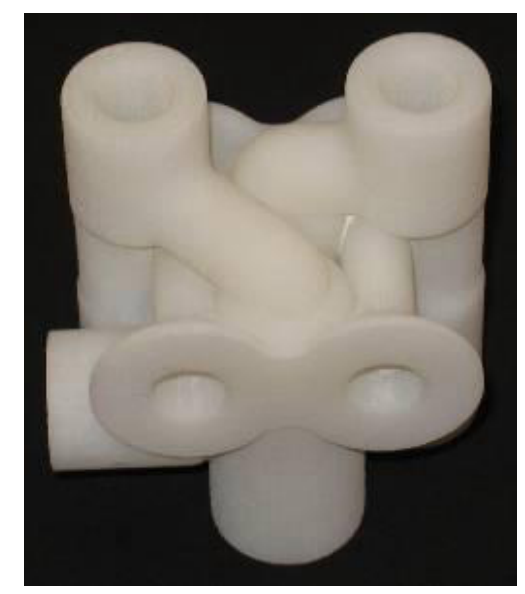

Figure 3. Pièce complexe réalisée en FA par Volum-e.

Par ailleurs, l'autre principal intérêt de la FA est la production en masse de pièces personnalisées. Cela serait trop onéreux pour les industriels de contrôler ces pièces en routine avec des techniques élaborées de type tomographie à rayons $\mathrm{X}$. En conséquence, des méthodes de contrôle de routine qualifiées, non-destructives et nonaltérantes, doivent être investiguées. Enfin, de par le principe de fabrication, couche par couche, les pièces présentent un effet escalier et sont rugueuses. Leur état de surface doit être caractérisé.

\subsection{Contrôles sur la machine}

Si la FA n'est pas une technologie nouvelle, son utilisation pour la réalisation de pièces fonctionnelles est récente. Or, les procédés ne sont pas encore suffisamment répétables pour être totalement fiables. En conséquence, les machines doivent être qualifiées. Cela peut être réalisé de deux façons différentes : soit en caractérisant toutes les composantes de la machine séparément, soit en réalisant un échantillon témoin que l'on caractérise ensuite. Cependant, caractériser toutes les composantes des machines nécessite d'instrumenter l'intérieur de la chambre de fabrication. D'une part, cela n'est pas forcément possible. D'autre part, cela est beaucoup plus compliqué que de réaliser un échantillon témoin. Par ailleurs, l'intérêt de ce dernier est de permettre non seulement l'évaluation et la vérification des performances de la machine (quantification de son exactitude, identification des sources d'erreurs...), de combiner toutes les erreurs de la machine mais également de comparer les machines entre elles.

\section{Conclusions}

Nous avons présenté dans ce papier, la fabrication additive (FA) ainsi que les besoins en métrologie et essais que nous avons identifiés spécifiquement pour cette technologie. Ces besoins se répartissent sur la matière première, sur le matériau, sur la pièce finie et sur la machine. Ces mesures et essais nécessitent d'être formalisés. A cet effet, des méthodologies de contrôle, des protocoles expérimentaux doivent être définis afin de valider la fiabilité des pièces réalisées en FA, ainsi que les machines de FA. Par ailleurs, les procédures de qualification dédiées à des pièces réalisées par des méthodes traditionnelles doivent être révisées pour s'adapter aux pièces réalisées en FA. Cela doit se faire dans le cadre de normes. Les organismes de normalisation UNM 920 en France et ISO TC 261 à l'international doivent poursuivre leur travail en ce sens.

\section{Références}

1. A-F. Obaton, A. Bernard, G. Taillandier, J-M. Moschetta, RFM, 37 (2015). 